\title{
Ações do núcleo de segurança do paciente em um hospital geral de ensino: relato de experiência de um estágio extracurricular
}

\author{
Actions of the patient safety nucleus in a large general teaching hospital: experience report \\ of an extracurricular internship
}

Acciones del núcleo de seguridad del paciente en un gran hospital general de enseñanza: informe de experiencia de una práctica extracurricular

Ana Tereza Cipriano de Oliveira ${ }^{1}$, Tarcisio Oliveira Silva ${ }^{2 *}$, Aldacy Gonçalves Ribeiro², Elisangela de Queiroz Oliveira², Aline Brandão Lima², Rogério Ribeiro².

\section{RESUMO}

Objetivo: Relatar experiências vivenciadas no estágio extracurricular no Núcleo de Segurança do Paciente em um hospital de grande porte de uma cidade da Bahia. Relato de Experiência: Como ponto de partida a estagiária se apropriou do conhecimento sobre os protocolos, rotinas, documentos, e sobre as metas internacionais de segurança do paciente que estavam em processo de implementação no hospital. A prática do estágio foi iniciada com as atividades de identificação do paciente, realizando ações em loco nos setores de internamento do hospital. Logo após realizou-se atividades nesses setores para analisar o carrinho de emergência, verificando se estes possuíam registro de conferência e checagem das medicações e insumos. Em seguida no centro cirúrgico, foi experienciado a admissão de pacientes e identificação das não conformidades para uma cirurgia segura. Considerações finais: Fazer parte do Núcleo de Segurança do Paciente, possibilitou à discente colocar em prática os conteúdos aprendidos em sala de aula, fortalecendo a relação ensino-aprendizagem e preparando-a para uma assistência de enfermagem com qualidade, tendo como foco a segurança do paciente. Sendo esse estudo uma contribuição para os estagiários da área de saúde que desejem desenvolver atividades nessa área de atuação.

Palavras-chave: Enfermagem, Segurança do paciente, Estágio clínico.

\begin{abstract}
Objective: Report of experiences lived in the extracurricular internship at the Patient Safety Center in a large hospital in a city in Bahia. Experience report: As a starting point, the trainee appropriated knowledge about the protocols, routines, documents and as international goals for patient safety that was being implemented in the hospital. The practice of the internship was created with the activities of patient identification, performing actions in loco in the sectors of hospitalization. Right after the activities were carried out in sectors to analyze the emergency cart, checking if they had a conference record and checking medications and supplies. Then, in the operating room, the admission of patients and the identification of nonconformities for safe surgery were tried. Final considerations: Being part of the Patient Safety Center, enabling the student to put into practice the content learned in the classroom, strengthening the teachinglearning relationship and preparing it for quality nursing care, with a focus on patient safety. This study being a contribution for health interns who wish to develop activities in this area.
\end{abstract}

Keywords: Nursing, Patient safety, Clinical stage.

${ }^{1}$ Centro Universitário Ruy Barbosa - Widen, Salvador - BA.

${ }^{2}$ Hospital Geral Roberto Santos. Salvador - BA. *E-mail: tosbahia@gmail.com

SUBMETIDO EM: 9/2019 | ACEITO EM: 10/2019 | PUBLICADO EM: 9/2020 


\section{RESUMEN}

Objetivo: Reporte de experiencias vividas en el internado extracurricular en el Centro de Seguridad del Paciente de un gran hospital de una ciudad de Bahía. Informe de experiencia: Como punto de partida, el pasante se apropió del conocimiento sobre los protocolos, rutinas, documentos y como metas internacionales para la seguridad del paciente que se estaba implementando en el hospital. La práctica del internado se creó con las actividades de identificación de pacientes, realizando acciones in loco en los sectores de hospitalización. Inmediatamente después se llevaron a cabo las actividades en los sectores para analizar el carro de emergencia, verificando si tenían registro de conferencias y revisando medicamentos y suministros. Luego, en quirófano, se intentó el ingreso de pacientes y la identificación de no conformidades para una cirugía segura. Consideraciones finales: Formar parte del Centro de Seguridad del Paciente, capacitando al alumno para poner en práctica los contenidos aprendidos en el aula, fortaleciendo la relación enseñanza-aprendizaje y preparándolo para una atención de enfermería de calidad, con enfoque en la seguridad del paciente. Este estudio es un aporte para los internos de salud que deseen desarrollar actividades en este ámbito.

Palabras clave: Enfermería, Seguridad del paciente, Estadia clínica.

\section{INTRODUÇÃO}

Na formação acadêmica do enfermeiro diversas são as etapas a serem cumpridas, o estágio é um dos momentos primordiais nesse processo, pois, é durante a sua realização que surgem as oportunidades para o aprendizado a partir de situações reais. Também é nesse momento que se é percebido o valor de uso de todos os conhecimentos teóricos já adquiridos (OLIVEIRA ILS, 2012).

O Programa de Estágio do Governo do Estado da Bahia oferece aos estudantes contrato com duração de um ano, contemplando atividades previstas no plano de estágio, que contribuem para o desenvolvimento pessoal e profissional dos participantes. O público-alvo do programa é composto pelos universitários regularmente matriculados em cursos superiores na modalidade presencial, que tenham concluído $50 \%$ da graduação. Dentre os setores ofertados pelo hospital em que o estudante atuará, o universitário faz a escolha pelo setor que deseja, dentre os setores ofertados está o Núcleo de Segurança do Paciente (NSP) (BAHIA, 2018).

Segundo a Resolução da Diretoria Colegiada (RDC) n. 36/2013 (ANVISA, 2013), o "Núcleo de Segurança do Paciente (NSP) é a instância do serviço de saúde criada para promover e apoiar a implementação de ações voltadas à segurança do paciente", consistindo em um componente extremamente importante na busca pela qualidade das atividades desenvolvidas nos serviços de saúde. Um dos objetivos específicos do NSP é promover e apoiar a implementação de iniciativas voltadas à segurança do paciente em diferentes áreas da atenção, organização e gestão de serviços de saúde (ANVISA, 2016).

Este núcleo deve ter como finalidade a promoção de uma cultura hospitalar voltada para a segurança dos pacientes, através de planejamento, desenvolvimento, controle e avaliação de processos assistenciais, a fim de garantir a qualidade dos mesmos em cada hospital. Além disso, o núcleo deve avaliar o grau de adesão da instituição de saúde para as práticas e recomendações emitidas por ele ou pelos órgãos, como Anvisa e Ministério da Saúde (BRASIL, 2014).

Existem áreas específicas como, controle de infecções hospitalares que apresentou uma tímida progressão. Considerando os vários riscos aos quais os pacientes no período de internação hospitalar estão submetidos, as Infecções Relacionadas à Assistência à Saúde (IRAS) destacam-se como as mais ameaçadoras, uma vez que possuem altos índices no cenário brasileiro, como também pela elevada morbimortalidade associada. Enquanto líder, o enfermeiro assume a responsabilidade da condução de mudanças e incorporação de novas tecnologias necessárias que favoreçam a qualidade e segurança do paciente no ambiente hospitalar (PRATES CG, et al., 2019; PINA E, et al., 2016; RADUENZ AC, et al., 2010). 
Como estratégia de atuação dos Núcleos de Segurança do Paciente, a Organização Mundial de Saúde (OMS) estabelece metas objetivando promover melhorias específicas na segurança do paciente por meio de estratégias que abordam aspectos problemáticos na assistência à saúde, apresentando soluções baseadas em evidências para esses problemas. São elas: (Meta 1) Identificar corretamente o paciente; (Meta 2) Melhorar a comunicação entre profissionais de saúde; (Meta 3) Melhorar a segurança na prescrição, no uso e na administração de medicamentos; (Meta 4) Assegurar cirurgias com local de intervenção, procedimento e paciente corretos; (Meta 5) Reduzir o risco de infecções associadas aos cuidados de saúde, com higienização das mãos; (Meta 6) Reduzir o risco de queda e prevenir lesões por pressão (WHO, 2007).

Essas metas objetivam efetivar condições básicas para a garantia de um ambiente livre de danos, favorecendo a condução de medidas pautadas em ações protocolares e de conhecimento de toda a equipe gestora e assistencial focadas em ações que garantam a segurança do paciente (WHO, 2007).

Pensando nisso, houve uma inquietação inicial da discente por existir poucos estudos relatando as vivências de universitários em estágios extracurriculares em segurança do paciente, surgindo a seguinte questão: quais as ações desenvolvidas por uma estagiária no Núcleo de Segurança do Paciente?

Assim, este estudo tem como objetivo: Relatar experiências vivenciadas no estágio extracurricular no Núcleo de Segurança do Paciente em um hospital de grande porte do município de Salvador - BA.

\section{RELATO DE EXPERIÊNCIA}

Trata-se de um estudo descritivo, apresentado como relato de experiências de vivências em um estágio extracurricular em enfermagem, vinculado ao Programa Partiu Estágio no período de 2 de maio de 2018 à 2 de abril de 2019, em um hospital de ensino e de grande porte da capital baiana, tendo como lócus de atuação o Núcleo de Segurança do Paciente (NSP).

Após a seleção e aprovação no Programa Partiu Estágio, a estagiária, autora desse estudo foi lotada no NSP de um hospital de ensino de grande porte do estado da Bahia. Como ponto de partida para todos os estagiários que compõem a equipe do NSP da referida instituição, esta foi apresentada à equipe que compõe o setor para que a mesma desse início a apropriação do conhecimento sobre os protocolos, rotinas e documentos essenciais para o monitoramento das metas de segurança do paciente.

Para cada uma das metas internacionais de segurança do paciente: 1) Identificar corretamente o paciente; 2) Melhorar a comunicação entre profissionais de Saúde; 3) Melhorar a segurança na prescrição, no uso e na administração de medicamentos; 4) Assegurar cirurgia em local de intervenção, procedimento e paciente corretos; 5) Higienizar as mãos para evitar infecções; 6) Reduzir o risco de quedas e úlceras por pressão, a equipe do NSP implementa ações, e os estagiários que integram este setor participaram de todo esse processo junto com a equipe (WHO, 2007).

A estagiária iniciou suas atividades no NSP atuando em estratégias que envolveram o cumprimento da meta número 1, identificar corretamente o paciente e meta de número 5 , higienizar as mãos para evitar infecções. Realizando visitas diárias em loco em alguns setores do hospital (enfermarias, Unidades de terapia intensiva - UTI's e emergências) nas quais verificava o uso da pulseira de identificação, e o uso da placa de identificação do leito de cada paciente, assim como a disponibilidade de insumos essenciais para a higiene das mãos (dispensadores de álcool em gel, sabão, pia e papel toalha em cada setor).

Diante de inconformidades atuava como agente ativo no processo de educação em saúde, estimulando reflexões na equipe multidisciplinar sobre a importância da adesão as metas de segurança do paciente, promovendo o fortalecimento dessa cultura. Esses dados coletados diariamente nestas visitas eram lançados em uma planilha Excel, e posteriormente tratados, auxiliando a elaboração de informações que dão origem aos indicadores de qualidade de adesão as metas de segurança do paciente. 
Segundo Hoffmeister LV e Moura GMSS (2015), frequentemente os serviços de saúde utilizam diferentes maneiras de identificação do paciente, como placas, pulseiras, adesivos nas roupas e crachás. Com a instituição da Política Nacional de Segurança do Paciente (PNSP), foi despertada a necessidade da implementação de estratégias de identificação com padrões específicos que garantam a segurança do paciente de acordo com os riscos que estes estão submetidos.

Apesar de não haver um padrão universal a instituição em estudo adotou para uso nas pulseiras e placas nos leitos: nome do paciente, cadastro hospitalar, data de admissão, data de nascimento, alergias. Além de identificação por cores (adesivos) para riscos de queda, flebite, lesão por pressão, broncoaspiração, trombose venosa profunda, nutricional e infecções.

Considerando a implementação de ações para alcance da meta 3, "Melhorar a segurança na prescrição, no uso e na administração de medicamentos", as ações da estagiária estiveram voltadas a realizar visitas nas unidades assistenciais para analisar o carrinho de emergência, verificando se estes possuíam registro de conferência e checagem das medicações e insumos.

Caso o carrinho de emergência estivesse lacrado era considerado o prazo de um mês para nova conferência. Também monitorava-se o desfibrilador, atentando se este equipamento estava adaptado a tomada, assim como a integridade do lacre do carrinho. Ainda sobre a abordagem da meta 5 , a estagiária atentava-se para a data da punção e sinais de infecção de acessos venosos periféricos e central do paciente, sinalizando prováveis datas de troca.

Llapa-Rodriguez EO, et al. (2017), na análise da adesão aos itens de segurança do paciente em uma UTI na cidade de São Paulo, descreve essa atuação como preocupante, indesejada e sofrível, por afetar diretamente a qualidade da assistência prestada. Na vivência da estagiária, esta também pôde observar o baixo conhecimento sobre as ações do NSP. Assim, para a popularização do conhecimento começou-se a discutir sobre as metas de segurança do paciente, eventos adversos e a importância do preenchimento da ficha de notificação, como notificar e para qual setor reportar.

Alguns setores do hospital, como o centro cirúrgico, ambulatório e Centro de Hemorragia Digestiva (CHD), fazem uso do checklist de cirurgia segura ou procedimento seguro, através de formulários específicos, e a estagiária teve a oportunidade de atuar no monitoramento destes instrumentos.

Esses eram recolhidos dos respectivos setores e os dados extraídos dos mesmos eram lançados em uma planilha Excel para a produção de gráficos mensais mensurando a adesão ao instrumento, fortalecendo a meta de número 4 , assegurar cirurgia em local de intervenção, procedimento e paciente corretos.

Essa prática de uso de checklist em procedimentos cirúrgicos é apontada como minimizador da dependência da memória, diminuindo erros, sendo traduzidos como qualidade da assistência prestada e qualificando processos e trazendo grandes contribuições na organização do processo de trabalho. Esses formulários uma vez monitorados podem contribuir no processo de educação permanente, trazendo benefícios à saúde dos pacientes (ALPENDRE FT, et al., 2017).

Uma outra experiência envolvendo a meta 4, foi a vivência no Centro cirúrgico para atuação na área de transferência, admitindo pacientes, observando a existência de todos os documentos em prontuário, necessários para a realização de cirurgia, realizando o preenchimento da ficha de avaliação perioperatória, e por fim, lançando estas informações no livro de registro da área de transferência para posterior produção dos indicadores de não conformidades.

Este livro contém as seguintes informações: data, registro, unidade de origem, nome do paciente, especialidade cirúrgica, procedimento a ser realizado, sala para onde o paciente realizará o procedimento e as não conformidades (banho, próteses tricotomia, lateralidade e demarcação, uso da pulseira de identificação, exames laboratoriais e de imagem, termo de consentimento assinado, avaliação perioperatória e avaliação anestésica). 
A estagiária também acompanhou a rotina da enfermeira administrativa do Centro Cirúrgico, que tem como algumas atribuições: seguir o roteiro do mapa cirúrgico, verificando a disponibilidade de salas operatórias e equipes cirúrgicas, realizar passagem de plantão entre os setores, quando necessário, registrar atraso nas cirurgias eletivas e suspensão cirúrgica, realizar o controle de temperaturas nas salas operatórias e registros no livro de ocorrências. Ainda foi possível vivenciar a rotina da enfermeira da sala operatória, tendo como atribuições: monitorização do paciente, lateralização na mesa cirúrgica, passagem de sonda vesical de demora e demais procedimentos técnicos da equipe de enfermagem. Além de aplicar o checklist de cirurgia segura, preencher a segunda parte (transoperatório) na ficha perioperatória, e administrar antibióticos profiláticos, quando prescrito.

\section{DISCUSSÃO}

Como primeira aproximação da estagiária ao NSP, foi priorizada a meta de identificação. No cotidiano do hospital pôde ser observado a adoção de diferentes maneiras de identificar os pacientes como pulseiras, placas nas cabeceiras, adesivos nas roupas e crachás. E o NSP possui a rotina de verificação da adesão a essas formas de identificação, no entanto com pouca efetividade. E apesar de serem poucos os estudos que abordem especificamente o tema de identificação do paciente, já é possível perceber uma preocupação mundial em relação a essa prática (HOFFMEISTER LV e MOURA GMSS, 2015).

Um estudo, realizado por Tase TH, et al. (2013) em uma maternidade no município de São Paulo avaliou pulseiras de identificação e definiu como prioritária quatro qualificadores: o nome completo da mãe, o número de atendimento da mãe ou do recém-nato, o tipo de internação e o código de barras legíveis. Com a aplicação de um impresso para monitoramento do uso da pulseira de identificação no hospital aqui estudado, percebeu-se a necessidade de qualificar pacientes e acompanhantes para que se evite a retirada da pulseira de identificação, o que favoreceriam erros.

Para monitoramento da higienização das mãos pelo NSP, é utilizado instrumento protocolar que mensura o número de oportunidades para a realização dessa ação, e a adesão ou não do funcionário a este cuidado básico. Bem como, a qualidade da higienização realizada (tempo de duração, quantidade de produto utilizado, uso de luvas, joias, esmaltes, unhas postiças, etc). Essa técnica validada pela OMS é aplicada nas instituições de saúde em forma de "check list", elaborado em parâmetros do Ministério da Saúde brasileiro (PRIMO MGB, et al., 2010).

As ações desenvolvidas no estágio envolvendo o protocolo de higienização das mãos, foi desde a lavagem das mãos até a não utilização de adornos, esse monitoramento é realizado através de um impresso aplicado nos setores do hospital, com atividades de educação em saúde realizada com os profissionais de saúde e acompanhantes dos pacientes, ou até mesmo com o próprio paciente, evitando assim infecções cruzadas, podendo diminuir os dias de internação do paciente no hospital. Observou-se ainda que a checagem dos itens (pia funcionante, dispensador de álcool em gel, sabão e dispensador de papel toalha) influenciam na adesão desse protocolo de higienização das mãos. Pensando na perspectiva de adesão a esta meta, foi disponibilizado aos profissionais/trabalhadores em saúde um dispositivo denominado guarda-adornos, com o intuito de estimular a guarda de joias e enfeites durante a jornada de trabalho, reduzindo assim os índices de infecção.

Com relação a meta de cirurgia segura pode-se perceber que a implementação do checklist de cirurgia segura e o monitoramento das não conformidades reduzem em números consideráveis eventos adversos, preservando assim a segurança do paciente cirúrgico. Dessa forma, em estudo multicêntrico Haynes AB, et, al. (2009), mostrou uma redução de $36 \%$ das complicações e $47 \%$ da mortalidade em pacientes cirúrgicos após a implantação do checklist de cirurgia segura.

O checklist é uma lista de verificação que contém itens para uso da equipe assistencial e de gestão, que quando checados evita-se erros em procedimentos por tratar-se de ação protocolar validada pela instituição e embasado em evidências científicas, garantindo a qualidade do processo de trabalho desenvolvido pela equipe e a segurança do paciente (CARVALHO R e BIANCHI ERF, 2016). 
O protocolo de cirurgia segura quando aplicado no serviço de saúde, inicia-se na admissão do paciente na sala de transferência, onde é preenchida a ficha de avaliação pré-operatória e as não conformidades, e após a aplicação do checklist de cirurgia segura no transoperatório. A vivência desse processo permitiu a construção do conhecimento, favorecendo a troca de saberes. Vale destacar a extrema relevância das várias categorias profissionais na relação com a equipe de enfermagem para o alcance da qualidade e segurança do paciente. Assim, espera servir como propulsor para novos estudos nesta temática.

\section{REFERÊNCIAS}

1. ALPENDRE FT, et al. Cirurgia segura: validação de checklist pré e pós-operatório. Rev. Latino-Am. Enfermagem, 2017; 25:e2907

2. ANVISA. Resolução de Diretoria Colegiada - RDC № 36, de 25 de Julho de 2013. Ministério da Saúde - MS. Agência Nacional de Vigilância Sanitária - ANVISA, 2013.

3. ANVISA. Núcleos de Segurança do Paciente. Portal da Anvisa, 2016.

4. BAHIA. EDITAL № 001/2018-DDE/SRH/SAEB. Secretaria da Administração do Estado da Bahia - SAEB. CNPJ: 13.323.274/0001-63. Bahia, 2018.

5. BRASIL. Programa Nacional de Segurança do Paciente (PNSP). Portal do Ministério da Saúde, 2013.

6. BRASIL. Documento de referência para o Programa Nacional de Segurança do Paciente. Ministério da Saúde; Fundação Oswaldo Cruz; Agência Nacional de Vigilância Sanitária. Brasília: Ministério da Saúde, 2014.

7. BRASIL. Ministério de Educação. Diretriz para Implantação dos Núcleos e Planos de Segurança do Paciente nas Filiais Ebserh, [S. L.], 2014.

8. CARVALHO R, BIANCHI ERF. Enfermagem em Centro Cirúrgico e Recuperação. 1ª ed. $2^{\mathfrak{a}}$ edição revisada e atualizada, BEUWEI: Manoele, 2016.

9. HAYNES AB, et al. A surgical safety checklist to reduce morbidity and mortality in a global population. $\mathrm{N}$ Engl J Med. 2009; 360(5):491.

10. HOFFMEISTER LV, MOURA GMSS. Uso de pulseiras de identificação em pacientes internados em um hospital universitário. Rev. Latino-Am. Enfermagem, jan.-fev. 2015; 23(1):36-43

11. LLAPA-RODRIGUEZ EO, et al. Assistência segura ao paciente no preparo e administração de medicamentos. Rev Gaúcha Enferm. 2017; 38(4): e2017-0029.

12. PINA E, et al. Infeções associadas aos cuidados de saúde. In: SOUSA, P., MENDES, W., orgs. Segurança do paciente: conhecendo os riscos nas organizações de saúde [online]. Rio de Janeiro: Editora FIOCRUZ, 2014. 1: 137-158. 452 p. ISBN: 978-85-7541-595-,

13. PRATES CG, et al. Núcleo de segurança do paciente: o caminho das pedras em um hospital geral. Revista Gaúcha de Enfermagem, 2019; 40(esp):e20180150.

14. PRIMO MGB, et al. Adesão à prática de higienização das mãos por profissionais de saúde de um Hospital Universitário. Rev Eletrônica de Enferm. 2010;12(2):266-71.

15. RADUENZ AC, et. al. Cuidados de enfermagem e segurança do paciente: visualizando a organização, acondicionamento e distribuição de medicamentos com método de pesquisa fotográfica. Rev. Latino-Am. Enferm., Ribeirão Preto, v. 18, n. 6, p. 1045-1054, nov./ dez. 2010.

16. 16 OLIVEIRA ILS. A importância do estágio na formação profissional. Portal da educação, 2012.

17. TASE TH, et al. Identificação do paciente nas organizações de saúde: uma reflexão emergente. Rev Gaúcha Enferm. 2013;34(2):196-200.

18. WHO. Patient Safet Solutions Preamble. World Health Organization. May, 2007. 